\title{
Utility-based Resource Allocation with Bipartite Matching in OFDMA-based Wireless Systems
}

\author{
Kan Zheng ${ }^{1}$, Wei $\mathrm{Li}^{1}$, Fei Liu ${ }^{1}$ and Wei Xiang ${ }^{2}$ \\ ${ }^{1}$ Wireless Signal Process\& Network Lab, Key Lab of Universal Wireless Communications, Ministry of Education, \\ Beijing University of Posts\& Telecommunications \\ Beijing, P. R. China, 100088 \\ ${ }^{2}$ Faculty of Engineering and Surveying \\ University of Southern Queensland \\ Toowoomba, QLD 4350, Australia \\ *Correspondingauthor: Kan Zheng \\ [e-mail: kzheng@ieee.org]
}

Received June 8, 2012; revised June 28, 2012; revised July 10, 2012; accepted July 13, 2012;

published August 30, 2012

\begin{abstract}
In order to efficiently utilize limited radio resources, resource allocation schemes in OFDMA-based wireless networks have gained intensive attention recently. Instead of improving the throughput performance, the utility is adopted as the metric for resource allocation, which provides reasonable methods to build up the relationship between user experience and various quality-of-service (QoS) metrics. After formulating the optimization problem by using a weighted bipartite graph, a modified bipartite matching method is proposed to find a suboptimal solution for the resource allocation problem in OFDMA-based wireless systems with feasible computational complexity. Finally, simulation results are presented to validate the effectiveness of the proposed method.
\end{abstract}

Keywords: OFDMA, bipartite matching, utility. 


\section{Introduction}

Due to its efficient utilization of available frequency bandwidth and robustness to frequency-selective fading environments, orthogonal frequency division multiplexing access(OFDMA) has been accepted as a multiple access scheme in the third-generation (3G) long-term evolution (LTE) networks and its advancement (LTE-Advanced) for downlink transmission[1]. In OFDMA-based systems, users are multiplexed in frequency by means of a scheduler which assigns the sub-channels, consisting of a number of subcarriers and time slots, to different users according to predefined resource allocation schemes.

The majority of resource allocation mechanisms aim at maximizing the throughput or spectralefficiency, while keeping certain quality-of-service (QoS) metrics atacceptable levels [2][3][4][5][6]. However, the satisfaction of the userdoes notjustdependon a single QoS metric, e.g., throughput. Throughput maximization leads to performance whichis usually not optimal with respect to user satisfactionfor multimedia applications.Utility theory provides reasonable methods to formulate the relationship between user experience and various QoS metrics. Utility functions can be used to quantify the satisfaction of using radio resources. Therefore, utility-based resource allocation schemes can better meet the service requirements of users'applications.In the past few years, utility-based resource optimization problems are emerging in the wireless research community. In [7], the utility-based resource allocation problem is studied to maximize the total utility of the system with only a single best-effort (BE) application, where several forms of the utility function for BE are used. Then, a scheduler for the OFDM wireless network with mixed real-time and non-real-time applications is designed to adaptively adjust the resources including sub-channels and transmit power based on the utility functions [8]. Unlike the objective of utility maximization, the authors in [9] propose an adjustable fairness scheduler that allows the specification of a desired fairness constraint, where the utility of a user is measured by means of the fairness utility functions.

Recently, theoretical graph tools are being used to solve the problem of sub-channel allocation in an OFDMA wireless network. Most of the existing resource allocation methods based upon graph tools fall under two categories, i.e., 1) graph coloring, [10][12][13] and 2) Maximum Weighted Matching (MWM) in a weighted bipartite graph[14][15]. In this paper, we focus on the latter one, which is relatively less well-investigated than the former. After graph theoretical formulation, graph algorithms can be used to find optimal/suboptimal solutions for resource allocation problems. For example, the Kuhn-Munkres (KM) algorithm can be used to find the maximum assignment [13], i.e., the optimal solution to the maximum weight sum problem in a bipartite graph, which is equivalent to the solution to the maximum sum problem in resource allocation. In order to decrease the computational complexity, a modified sub-optimal min-sum belief propagation algorithm is used to implement MWM when solving the resource optimization problem [14]. Compared to MWM-based scheduling, a new class of channel-aware polling service-based scheduling schemes are proposed and can achieve comparable delay performance, but with significantly reduced computational complexity [15]. In [16], the utility-based resource scheduling problem is formulated into an MWM one and solved with a suboptimal algorithm, which improves the fairness of resource allocation.

In this paper, the problem of allocating sub-channels in an OFDMA network is modeled as finding a suitable matching in a bipartite graph. Firstly, we construct a bipartite graph, where one subset is the user set while the other subset is the subchannel set. Then, this bipartite graph 
is expanded by copying its rows or columnsto form anew bipartite graph, which can be used to solve resource allocation problems, where more than one sub-channel can be assigned to a user. Different from classic resource allocation mechanisms based on throughput or spectral efficiency, the utility of the user with the given service is chosen as the metric to determine which sub-channels are assigned to which user with the specified objective. The utility is used as the initial weight of the edge in the bipartite graph. However, due to the non-linearity of utility functions, these weights have to be modified with the proposed method when applying the well-known KM algorithm to find MWM in the expanded bipartite graph. Finally, our simulation results demonstrate the effectiveness of the proposed scheme in terms of the utility performance.

The remainder of this paper is organized as follows. Section 2 gives a brief description of the system model and formulates the channel allocation problem in OFDMA networks. A utility-based channel allocation scheme for OFDMA networks is proposed in Section 3. In Section 4, simulation results are presented. Finally, Section 5 concludes this paper

\section{Model Description}

Consider an OFDMA system with $N$ users on downlink transmission, and $M$ sub-channels available for resource allocation on a subframe-by-subframe basis. The base station can only transmit to a single user over each sub-channel. According to Shannon's theorem, the maximum achievable data rate on sub-channel $m$ assigned to user $n$ depends on the allocated bandwidth and its channel quality in terms of SNR, i.e.,

$$
\eta_{n, m}=W \log _{2}\left(1+\frac{L_{n} \beta_{n, m} P_{n, m}}{\sigma^{2}}\right), 1 \leq m \leq M, 1 \leq n \leq N,
$$

where $W$ is the sub-channel bandwidth, $L_{n}$ is the path loss attenuation factor from the base station to user $n, P_{n, m}$ and $\beta_{n, m}$ are the transmit power and the instantaneous channel gain from the base station to user non sub-channel $m$, respectively, and $\sigma^{2}$ is the noise power of the additive white Gaussian noise (AWGN). For simplicity, the transmit power is made the same for all the sub-channels, i.e., $P_{n, m}=P_{T}, 1 \leq m \leq M, 1 \leq n \leq N$.

Let the binary matrix $\mathbf{A}=\left\{a_{n, m} \mid a_{n, m} \in\{0,1\}\right\}_{N \times M}$ represent channel allocation betweenusers, where $a_{n, m}=1$ indicatesthat subchannel $m$ isassigned to usern, and $a_{n, m}=0$ otherwise. The radio resource allocation problem is to find $\mathbf{A}$ such that a pre-defined objective function is optimized with given resource constraints. A utility function is used as an effective trade-off between spectral efficiency and fairness of network resource allocation. The utility function maps the network resource use or performance criteria into a real number that can be used as a metric to quantify the user's satisfaction. Usually the data rate is one of the most important factors to influence the satisfaction of the user. Therefore, the utility is assumed to be a function of the data rate in this paper. Then, we focus on utility-based resource allocation schemes with the objective of maximizing the total utility, i.e.,

$$
\max \sum_{n=1}^{N} f_{n}\left(\sum_{m=1}^{M} a_{n, m} \eta_{n, m}\right)
$$

where $f_{n}(\bullet)$ is the utility function of user $n$.

In general, most applications can be classified into two types based on the characteristics of the utility function. The former is usually called as elastic applications, corresponding to 
traditional data services, such as file transfer and e-mail. These services can adjust their transmission data rates gradually, resulting in a graceful degradation of user satisfaction. The elasticity of these services can be modeled as concave utility functions. The latter corresponds to delay and rate sensitive services, such as streaming video and audio services. These applications are less elastic than the former. The elasticity of these services can be modeled by using sigmoidal-like utility functions. For the sake of analysis, two example utility functions for these two types of applications are given below

$$
\begin{gathered}
f_{1}(x)=\frac{\log _{2}\left(a_{1} \frac{x}{R_{\max }}+b_{1}\right)}{\log _{2}\left(a_{1}+b_{1}\right)} \\
f_{2}(x)=\frac{1}{1+e^{-a_{2}\left(\frac{x}{R_{\max }}-b_{2}\right)}}
\end{gathered}
$$

where $x$ represents the achievable data rate, $R_{\max }$ is the maximum transmission data rate of a user, $a_{1}, a_{2}, b_{1}$ and $b_{2}$ are the normalization parameters of the function. For simplicity, it is assumed that these parameters remain invariant for the users with the same type of applications in this paper.

\section{Proposed Resource Allocation Scheme with Bipartite Matching}

As mentioned above, sigmoidal-like utility functions are used for delay and rate sensitive services applications as well as data services with a concave utility function. This results in the non-convexity of the system. Hence, the optimization problem in (2) becomes non-convex, which is usually difficult to solve. Therefore, we propose a graph-based scheme to tackle the channel allocation problem in OFDMA wireless networks, which turns the optimization problem in (2) into finding the right matching in a bipartite graph.

\subsection{Construct the bipartite graph}

Based on a given network topology, we can first construct a bipartite graph $\mathbf{G}=(\mathbf{V}, \mathbf{E})$ with vertex set $\mathbf{V}$ and edge set $\mathbf{E}$.In this bipartite graph, all the vertices can be divided into two disjoint subsets. One subset of $\mathbf{V}$ is the user set $\mathbf{U}=\left\{u_{1}, u_{2}, \ldots, u_{N}\right\}$, whereas the other subset is the subchannel set $\mathbf{S}=\left\{s_{1}, s_{2}, \ldots, s_{M}\right\}$, where $n=1,2, \ldots, N$ indicates the $n$th user and $m=1,2, \ldots, M$ denotes the $m$ th sub-channel. It is evident that $\mathbf{V}=\mathbf{U} \cup \mathbf{S}$ and $\mathbf{U} \cap \mathbf{S}=\varnothing$.If every edge in $\mathbf{E}$ has one endpoint in $\mathbf{U}$ and the other in $\mathbf{S}$, the graph $\mathbf{G}=(\mathbf{V}, \mathbf{E})$ is called a bipartite graph, which can also be denoted by $\mathbf{G}=(\mathbf{U}, \mathbf{S}, \mathbf{E})$.Each edge in this graph is labeled as $\left(u_{n}, s_{m}\right) \in \mathbf{E}$, where $u_{n} \in \mathbf{U}$ and $s_{m} \in \mathbf{S}$. A subset of the edges, $\mathbf{M} \subseteq \mathbf{E}$, is called a matching if no two edges in $\mathbf{M}$ share common endpoints. A perfect matching is a matching which matches all vertices of the graph. That is, every vertex of the graph is incident to exactly one edge of the matching. Each edge $\left(u_{n}, s_{m}\right)$ is associated with a weight $w_{n, m}$, which is determined by its utility function. Fig. 1 illustrates a weighted bipartite graph. 


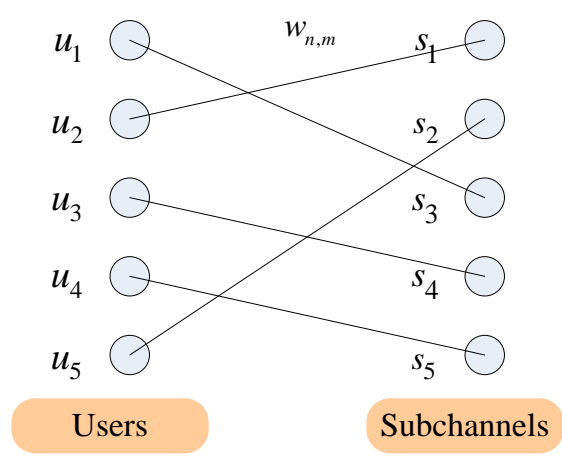

Fig. 1. Example of a weighted bipartite graph.

\subsection{Kuhn-Munkres Algorithm for MWM}

For a given matching $\mathbf{M}$ and edge $\left(u_{n}, s_{m}\right), c_{n, m}$ is an indicator which is equal to 1 if $\left(u_{n}, s_{m}\right)$ is in the matching $\mathbf{M}$ and $c_{n, m}=0$ otherwise. Let $\mathbf{C}=\left\{c_{n, m} \mid c_{n, m} \in\{0,1\}\right\}_{N \times M}$ be the permutation matrix corresponding to perfect matches. Given a weighted bipartite graph, Maximum Weighted Matching (MWM) finds that the optimum matching whose sum of weights is the largest, as determined by the following

$$
\mathbf{C}^{*}=\arg \max _{\mathbf{C} \in \mathbf{P}} \sum_{n=1}^{N} \sum_{m=1}^{M} c_{n, m} w_{n, m}
$$

where $\mathbf{P}$ denotes the set of permutation matrices. Assume that each user can only occupy one sub-channel, i.e., $\sum_{m=1}^{M} c_{n, m}=1$, and $w_{n, m}=f_{n}\left(\eta_{n, m}\right)$, the optimization problem shown in (2) is clearly equivalent to the MWM problem in (5). As a result, the resource assignment problem is turned into finding the optimum matching in the weighted bipartite graph.

For a bipartite graph $\mathbf{G}=(\mathbf{U}, \mathbf{S}, \mathbf{E})$, if the cardinalities of $\mathbf{U}$ and $\mathbf{S}$ are identical, i.e., $N=M$, this bipartite graph is symmetric or asymmetric otherwise. Optimality is guaranteed for symmetric graphs. However, usually the number of users is not equal to that of sub-channels. Thus we can expand the asymmetric graph with additional $|M-N|$ edges of zero weight so as to construct a symmetric graph. The extra nodes are added to the set of either the users or sub-channels that has a less number of nodes. It has been proved that the Kuhn-Munkres algorithm can always find the MWM for asymmetric bipartite graph [17]. Now, with the graph theoretical formulation, the optimal solution to the radio resource allocation problem can be solved by the KM algorithm described in detail as follows.

\section{Step 1: Label initialization.}

This algorithm starts with the following labels. $l\left(u_{n}\right)=\max _{s_{m} \in S} w_{n, m}$ for all the vertexes in $\mathbf{U}$ and $l\left(s_{m}\right)=0$ for all the vertexes in $\mathbf{S}$.

\section{Step 2: Construct the equality subgraph.}

The equality subgraph $\mathbf{G}^{\prime}$ is constructed in this step. The equality subgraph consists of the vertexessatisfying $l\left(u_{n}\right)+l\left(s_{m}\right)=w_{n, m}$ and the corresponding edges. Then, a maximum matching is found in the equality subgraph. If the maximum matching is a perfect matching, 
the process of the KM algorithm stops. Otherwise, it continues to Step 3.

Step 3: Adjustment of labels.

Let set $\mathbf{Q}$ be a vertex cover of $\mathbf{G}^{\prime}$, and let $\mathbf{X}=\mathbf{U} \cap \mathbf{Q}$ and $\mathbf{Y}=\mathbf{S} \cap \mathbf{Q}$. Now, the adjustment value is $\varepsilon=\min \left\{l\left(u_{n}\right)+l\left(s_{m}\right)-w_{n, m}\right\},\left(u_{n} \in \mathbf{X}, s_{m} \notin \mathbf{Y}\right)$. The label of the vertex in set $\mathbf{X}$ is decreased by $\varepsilon$, and the label of the vertex in set $\mathbf{Y}$ is increased by $\varepsilon$.

\section{Step 4: Repeat of step 2-3}

Steps 2 to 3 are repeated until the perfect matching is obtained.

\subsection{Modified KM Algorithm for Utility-based scheme}

In the bipartite graph $\mathbf{G}=(\mathbf{U}, \mathbf{S}, \mathbf{E})$, no two edges share common nodes, implying that no user can occupy more than one sub-channel. However, users are often allocated to more than one sub-channel in practical OFDMA wireless networks, i.e., $\sum_{m=1}^{M} a_{n, m} \geq 1$. In order to address this problem, a new bipartite graph $\tilde{\mathbf{G}}=(\tilde{\mathbf{U}}, \tilde{\mathbf{S}}, \tilde{\mathbf{E}})$ can be constructed by copying rows or columns of $\mathbf{G}=(\mathbf{U}, \mathbf{S}, \mathbf{E})$, and then solve the optimization problem by finding the MWM. Moreover, due to the nonlinearity of the utility functions, the weights cannot be summed up directly when the KM algorithm is applied in the search procedure. Hence, a modified KM algorithm is also proposed in this section.

Assume that the maximum number of sub-channels that a user can use is $K$. Based on $\mathbf{G}=(\mathbf{U}, \mathbf{S}, \mathbf{E})$, the nodes of the users and sub-channels are both replicated $(K-1)$ times to form the replicated node set, i.e., $\mathbf{U}_{r}$ and $\mathbf{S}_{r}$. Then, $\tilde{\mathbf{U}}=\mathbf{U} \cup \mathbf{U}_{r}$ and $\tilde{\mathbf{S}}=\mathbf{S} \cup \mathbf{S}_{r}$. The replicated sub-channel nodes are virtual ones and the actual total resources are thus not increased. Then, in the new bipartite graph $\tilde{\mathbf{G}}$, the weights of the edges that have one end node in $\mathbf{S}_{r}$ is set to zero, while the weights of the other edges are calculated by the proposed method in the following part. Fig. 2 presents an example of the weighted bipartite graph with replicated nodes. The dark gray nodes are virtual resource nodes, and the orange lines mean that weights of these edges are set to zero.

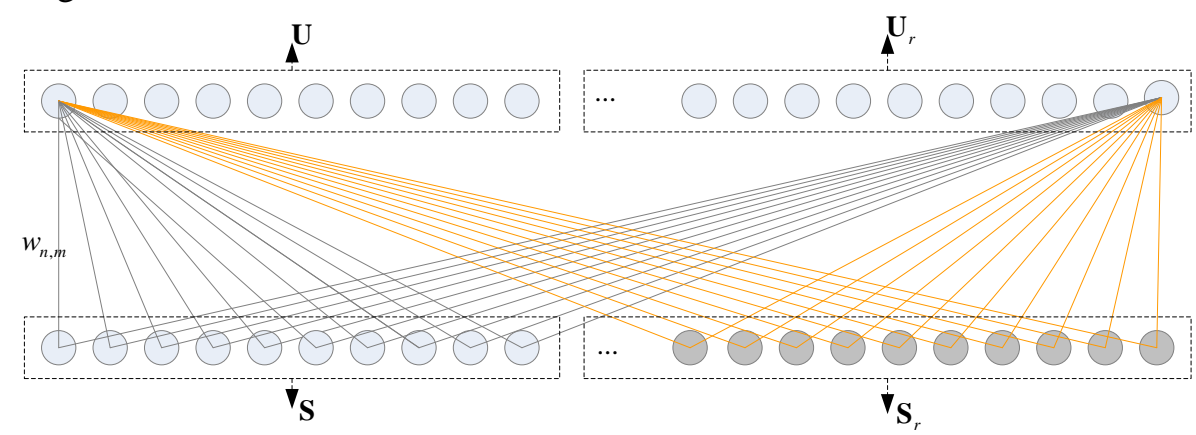

Fig. 2. A weighted bipartite graph with replicated nodes.

Then, applying the KM algorithm in searching the MWM in $\tilde{\mathbf{G}}$ can find the matching that gives the largest $\sum_{n} \sum_{m} c_{n, m} f_{n}\left(\eta_{n, m}\right)$ providing that the weight of the edges is still given 
by $f_{n}\left(\eta_{n, m}\right)$. However, the utility-based resource allocation problem is to find the optimal solution with largest $\sum_{n} f_{n}\left(a_{n, m} \sum_{m} \eta_{n, m}\right)$. Since the utility function is usually non-linear, these two problems cannot be regarded as equivalent. Therefore, we propose to modify the weights to find suboptimal solutions of the concerned resource allocation problem. Firstly, the weight of edge $(n, m)$ is initialized as

$$
\tilde{w}_{n, \bar{m}}^{\text {int }}=\left\{\begin{array}{l}
f_{n}\left(\eta_{n, m}\right), \text { if } \tilde{s}_{m} \in \mathbf{S} \\
0, \text { else }
\end{array}, 1 \leq n \leq K N, 1 \leq m \leq K M .\right.
$$

Then, the weight is adjusted by considering the increase of the utility if another sub-channel can also be assigned to the given user in the process of the KM algorithm. As stated before, each user has $K$ replicated copies in $\tilde{\mathbf{U}}$. Define the node set corresponding to user $n$ as $\tilde{\mathbf{U}}_{n}=\left\{\tilde{u}_{n}, \tilde{u}_{n+N}, \ldots, \tilde{u}_{n+(K-1) N}\right\}$, where $\tilde{\mathbf{U}}=\tilde{\mathbf{U}}_{1} \cup \tilde{\mathbf{U}}_{2} \cdots \cup \tilde{\mathbf{U}}_{N}$. When node $\tilde{u}_{n}$ in the user set is matched to node $\tilde{s}_{l}$ in the sub-channel set, the weight is modified as follows

$$
\tilde{w}_{n, m}=\left\{\begin{array}{l}
f_{n}\left(\eta_{n, m}+\eta_{n, l}\right)-f_{n}\left(\eta_{n, l}\right), \text { if } m \neq l, \tilde{u}_{n} \in \tilde{\mathbf{U}}_{n}, \tilde{s}_{l} \in \mathbf{S} \\
\tilde{w}_{n, m}^{\text {int }}, \text { else }
\end{array} .\right.
$$

As shown in Fig. 3, the first original user node has been matched with the red node in the original resource set. Then, weights of the blue edges connected with the duplicated nodes of the first one need to be changed according to (6).

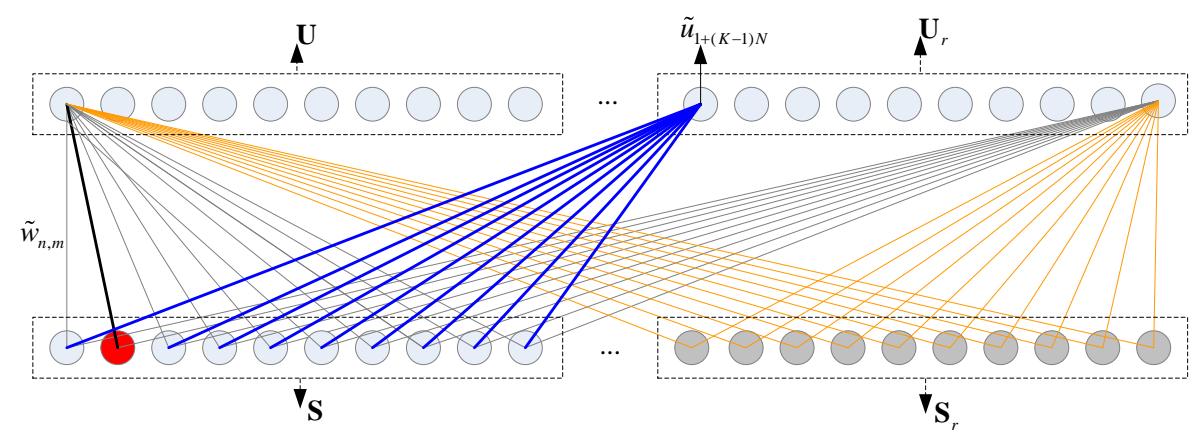

Fig. 3. Example of weight modification in the bipartite graph.

If $\tilde{\mathbf{G}}=(\tilde{\mathbf{U}}, \tilde{\mathbf{S}}, \tilde{\mathbf{E}})$ is not a symmetric graph, it is expanded with $|K M-K N|$ edges of zero weight to forma symmetric graph, i.e., $\tilde{\mathbf{G}}^{\prime}$. Finally, the KM algorithm is used to search the MWM in $\tilde{\mathbf{G}}^{\prime}$.

\section{Simulation Results}

In this section, simulation results are presented to evaluate the performance of the proposed channel allocation method for the OFDMA wireless network. The main system parameters are summarized in Table 1. 
Table 1. Main parameters in the OFDMA wireless network.

\begin{tabular}{|c|c|c|}
\hline \multicolumn{2}{|c|}{ Parameter } & Value \\
\hline \multicolumn{2}{|c|}{ eNB transmit power } & $43 \mathrm{dBm} / 5 \mathrm{MHz}$ \\
\hline \multicolumn{2}{|c|}{ Macro cell shadowing standard deviation } & $8 \mathrm{~dB}$ \\
\hline \multicolumn{2}{|c|}{ eNB antenna gain } & $14 \mathrm{dBi}$ \\
\hline \multicolumn{2}{|c|}{ UE antenna gain } & $0 \mathrm{dBi}$ \\
\hline \multicolumn{2}{|c|}{ Noise figure } & $9 \mathrm{~dB}$ \\
\hline \multicolumn{2}{|c|}{ Noise power spectral density } & $-174 \mathrm{dBm} / \mathrm{Hz}$ \\
\hline \multicolumn{2}{|l|}{ Path loss } & $128.1+37.6 \log (R)(\mathrm{dB}), R$ in $k m$ \\
\hline \multirow{3}{*}{$\begin{array}{l}\text { Utility function for } \\
\text { the elastic service }\end{array}$} & $a_{1}$ & 50 \\
\hline & $b_{1}$ & 1 \\
\hline & $R_{\max }$ & 667 \\
\hline \multirow{3}{*}{$\begin{array}{l}\text { Utility function for } \\
\text { the rate-sensitive service }\end{array}$} & $a_{2}$ & 15 \\
\hline & $b_{2}$ & 0.6 \\
\hline & $R_{\max }$ & 667 \\
\hline
\end{tabular}

4.1 Scenario A : $M=N=4$

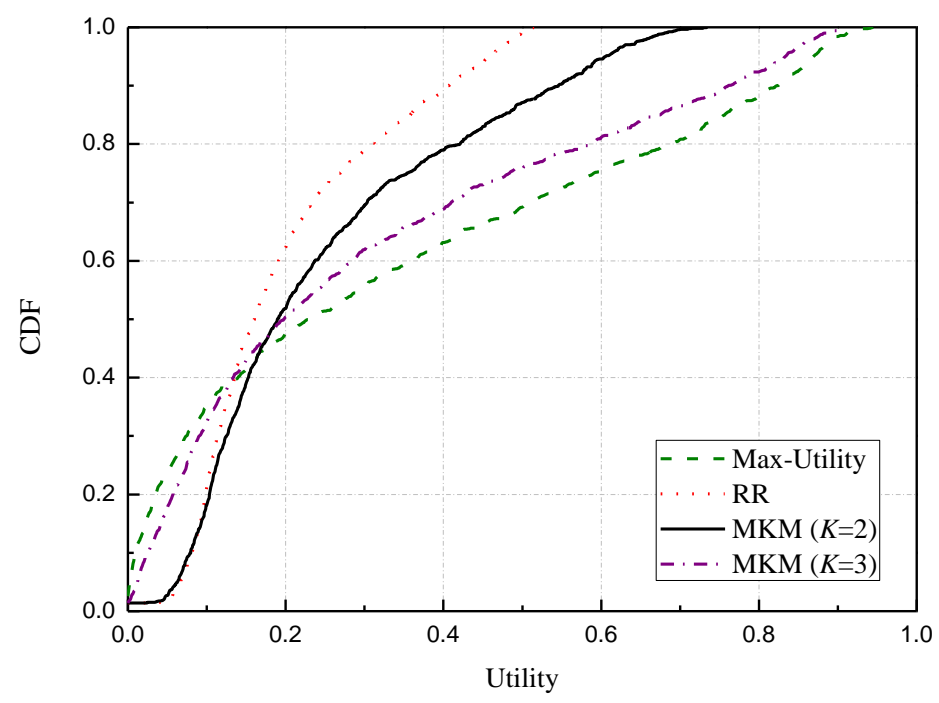

Fig. 4.CDF of utility with different resource allocation schemes.

Table 2. Utility performance with different resource allocation schemes $(M=N=4)$.

\begin{tabular}{|c|c|c|c|c|}
\hline & Optimal & $\operatorname{MKM}(K=2)$ & $\operatorname{MKM}(K=3)$ & RR \\
\hline \hline Average user utility & 0.3317 & 0.2501 & 0.2987 & 0.1999 \\
\hline $5 \%$ utility CDF & 0.0028 & 0.0632 & 0.0118 & 0.0671 \\
\hline
\end{tabular}

For simplicity, the number of users is assumed to be the same as that of sub-channels. Each user has the same elastic service function. The performance of the network with optimal channel allocation is also presented for comparison, i.e., the optimal solution of (2). This solution has prohibitive computational complexity. Therefore, we choose a small number of users and sub-channels $(M=N=4)$ so that exhaustive search is possible. Four different schemes are compared, i.e., 1) the optimal scheme, 2) the Round Rubin (RR) scheme, 3) the modified KM (MKM) with $K=2$, and 4) the modified KM (MKM) with $K=3$. Fig. 4 and Table 2 show 
the utility CDF and the average user utility of different channel allocation schemes. As far as utility efficiency is concerned, the optimal scheme based on exhaustive search is the best, while the RR scheme delivers the worst performance. The proposed MKM scheme is slightly worse compared with the optimal one but has substantially reduced computational complexity. With the increase of the number of the sub-channels that one user can occupy, i.e., from $K=2$ to $K=3$, the proposed MKM scheme has achieved better utility performance. The fairness performance comparison among these schemes is in the reverse order. Therefore, the proposed MKM scheme can achieve a good tradeoff between utility efficiency and fairness among users.

4.2 Scenario B : $M=N=10$

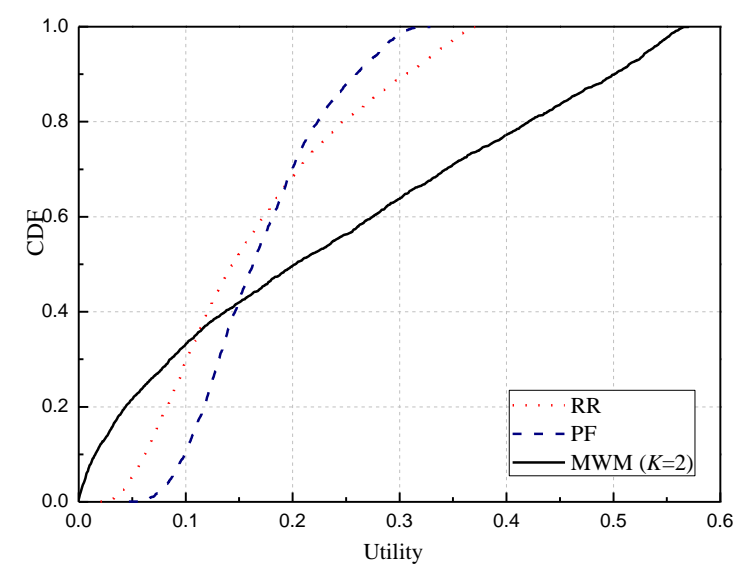

Fig. 5. CDF of utility with different resource allocation schemes with elastic services.

Table 3. Utility performance of different resource allocation scheme with elastic services $(M=N=10)$.

\begin{tabular}{|c|c|c|c|}
\hline & RR & PF & MKM $(K=2)$ \\
\hline \hline Average user utility & 0.1643 & 0.1709 & 0.2310 \\
\hline $5 \%$ utility CDF & 0.0492 & 0.0613 & 0.0063 \\
\hline
\end{tabular}

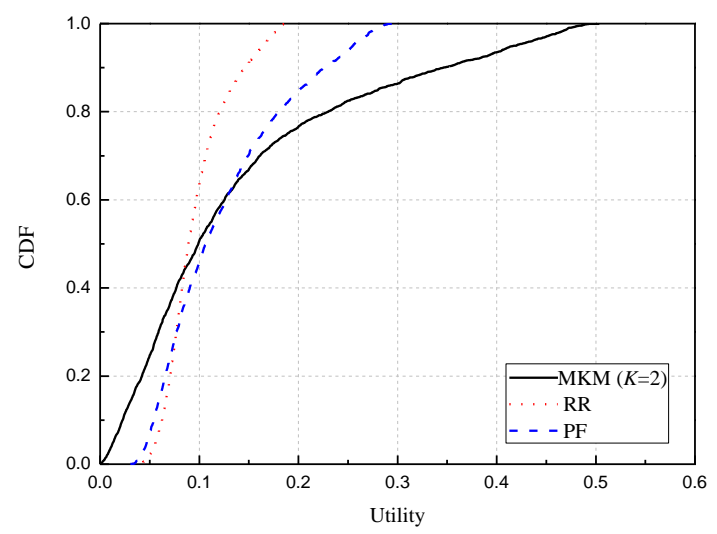

Fig. 6. CDF of utility with different resource allocation schemes with delay- and rate-sensitive service. 
Table 4. Utility performances of different resource allocation scheme with delay- and rate-sensitive service $(M=N=10)$.

\begin{tabular}{|c|c|c|c|}
\hline & RR & PF & MKM $(K=2)$ \\
\hline \hline Average user utility & 0.0967 & 0.1238 & 0.1403 \\
\hline $5 \%$ utility CDF & 0.0560 & 0.0471 & 0.0134 \\
\hline
\end{tabular}

Assume that each user has the same service function, i.e., either elastic or delay- and rate-sensitive. Due to the increase of the number of users and sub-channels, exhaustive search for the optimal scheme becomes infeasible. In this scenario, the performance of the proposed MKM scheme is compared with that with the two traditional scheduling schemes, i.e., RR and PF scheme. In Fig. 5 and Table 3, we present the CDF performance of utility and the average user utility of these channel allocation schemes with elastic service, respectively. As can be observed, the proposed MKM scheme outperforms the other schemes, and the RR scheme is worst in terms of the average utility. The average utility is increased by $35 \%$ with the MKM scheme as opposed to the PF scheme. However, the MKM scheme is worse than the others in fairness performance. As shown in Fig. 6 and Table 4, similar results are obtained when all the users are loaded with rate-sensitive services, but the RR and PF schemes show different comparative performance which is caused by the nonlinearity of the utility function.

4.3 Scenario C : $M=10, N=5$

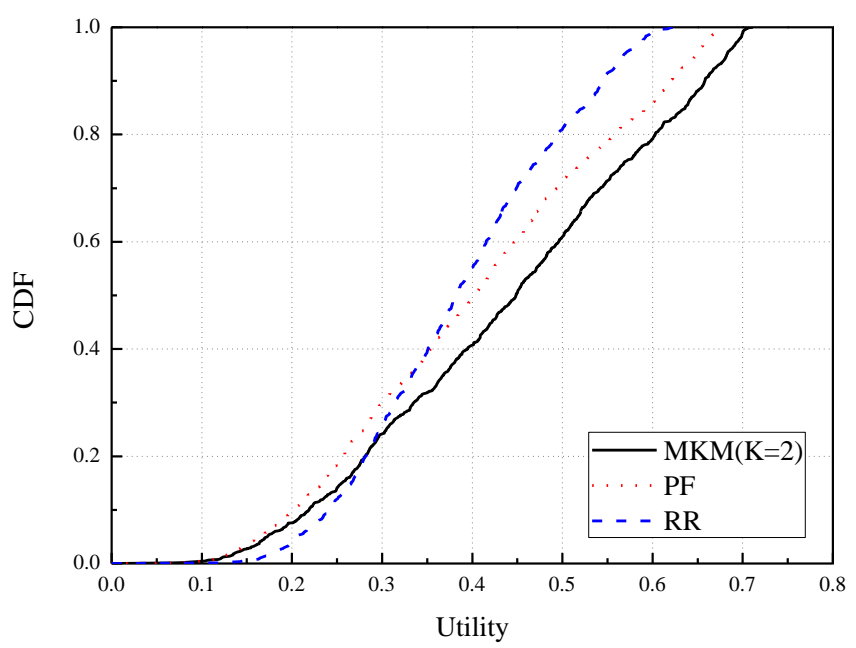

Fig. 7. CDF of utility with different resource allocation schemes.

Table 5. Utility performances of different resource allocation schemes $(M=10, N=5)$.

\begin{tabular}{|c|c|c|c|}
\hline & RR & PF & MKM $(K=2)$ \\
\hline \hline Average user utility & 0.3865 & 0.4036 & 0.4396 \\
\hline $5 \%$ utility CDF & 0.2059 & 0.1656 & 0.1731 \\
\hline
\end{tabular}

In this scenario, the number of the sub-channels is larger than the user number. The performance of the proposed MKM scheme and the two traditional ones are compared, while there are 10 sub-channels and 5 users with the elastic service. Fig. 7 gives the CDF of utility performance obtained by different schemes. The average user utility is also presented in Table 5. The MKM achieves higher utility performance than both of the RR and PF algorithms, but 
has worse fairness than the RR one. Compared with the simulation results in the 4.2 Scenario B, the utility performance in Scenario $\mathrm{C}$ is better. This attributes less users in Scenario C, so that one more sub-channel can be emploied by each of 5 users on average.

\section{Conclusion}

In this paper, the utility-based resource allocation problem in OFDMA-based networks is solved by a graphic scheme using bipartite matching. Firstly, a weighted bipartite graph is constructed, and the weights of the edges are determined by their utility function. Next, a modified KM algorithm is proposed to find the right matching in the constructed bipartite graph. Our simulation results demonstrate that the average utility performance is obviously improved in the proposed scheme in comparison with traditional non-optimal schemes with reasonable computational complexity.

\section{References}

[1] 3GPP TR 36.814, v2.0.1, "Further Advancements for E-UTRA, Physical Layer Aspects," August 2009. Article (CrossRef Link)

[2] Y. Zhang and K. B. Letaief, "Cross-Layer adaptive resource management for wireless packet Networks With OFDM Signaling," IEEE Trans. on Wireless Communications, vol. 5, no. 11, pp. 3244-3254, Nov. 2006. Article (CrossRef Link)

[3] A. Esmailpour, and N. Nasser, "Dynamic QoS-based bandwidth allocation framework for broadband Wireless Networks," IEEE Transactions on Vehicular Technology, vol. 60 no. 6, pp. 2690 - 2700, Jul. 2011. Article (CrossRef Link)

[4] L. Lei, C. Lin, J. Cai, and X. Shen, "Performance analysis of wireless opportunistic schedulers using stochastic Petri nets," IEEE Transactions on Wireless Communications, vol.8, no.4, pp.2076 - 2087 Article (CrossRef Link)

[5] X. Ge, K. Huang, C.-X. Wang, X. Hong, and X. Yang, "Capacity analysis of a multi-cell multi-antenna cooperative cellular network with co-channel interference," IEEE Transactions on Wireless Communications, vol. 10, no. 10, pp. 3298-3309, Oct. 2011. Article (CrossRef Link)

[6] I. Humar, X. Ge, L. Xiang, M. Jo, M. Chen, "Rethinking Energy - Efficiency Models of Cellular Networks with Embodied Energy,” IEEE Network Magazine, vol. 25, no. 3, pp. 40-49, Mar. 2011. Article (CrossRef Link)

[7] Z. Jiang, Y. Ge, and Y. Li, "Max-Utility wireless resource management for best-effort traffic", IEEE Transactions on Wireless Communications, vol. 4, no. 1, pp. 100-111, Jan. 2005. Article (CrossRef Link)

[8] M. Katoozian, K. Navaie, and H. Yanikomeroglu, "Utility-based adaptive radio resource allocation in OFDM wireless networks with traffic prioritization," IEEE Transactions on Wireless Communications, vol.8 , no. 1, pp. 66 - 71, Jan. 2009. Article (CrossRef Link)

[9] S. Schwarz, Mehlfuhrer, and C.M. Rupp, "Throughput maximizing multiuser scheduling with Adjustable Fairness," In Proc.of IEEE International Conference on Communications, Kyoto, Japan, pp.1-5, Jun. 2011.. Article (CrossRef Link)

[10] Jeannette Janssen, Kyriakos Kilakos, and Odile Marcotte, "Fixed preference channel assignment for cellular telephone systems", IEEE Transaction on Vehicular Technology, vol. 48, no. 2, pp. 533-540, Mar. 1999. Article (CrossRef Link)

[11] Y. Chen, N.Han, S. Shon, and J. Kim, "Dynamic frequency allocation based on graph coloring and local bargaining for multi-Cell WRAN System", in Proc. of Asia-Pacific Conference on Communications, Aug. 2006. Article (CrossRef Link)

[12] K. Zheng, Y. Wang, C. Lin, X. Shen, and J. Wang, "Graph-based interference coordination scheme in orthogonal frequency-division multiplexing access femtocell networks", IET Communications, vol. 5, no. 17, pp. 2533-2541, Nov. 2011. Article (CrossRef Link) 
[13] H. W. Kuhn, "The Hungarian Method for the Assignment Problem," Naval Research Logistic Quarterly, vol. 2, pp. 83-97, 1955. Article (CrossRef Link)

[14] P. Marilynn,G. Wylie, and P. Wang, "Cross Layer Design for OFDMA Systems Using the Beta-Min-Sum Belief Propagation Algorithm", in Proc. of IEEE Global Telecommunications Conference (Globecomm), pp. 5335-5340, Nov. 2007. Article (CrossRef Link)

[15] Y. Li, S. Mao, P. Agrawal, and F. Midkiff, "Low-Complexity Channel-Aware Scheduling for Multichannel Wireless Local Area Networks", in Proc. of IEEE Conference on Automation Science and Engineering, pp.133-138, Aug. 2008. Article (CrossRef Link)

[16] P. Marilynn, and P. Wang, "Utility-based Cross Layer Optimization for OFDMA Systems using the $\beta$-Min-Sum Belief Propagation Algorithm", in Proc. of Fourth International Conference on Broadband Communications, Networks and Systems, pp. 459-468, Sep. 2007. Article (CrossRef Link)

[17] H. W. Kuhn, "The Hungarian Method for the Assignment Problem", Naval Research Logistic Quarterly, vol. 2, pp. 83-97, 1955. Article (CrossRef Link)

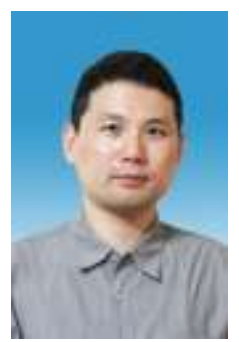

Kan Zheng (M'03-SM'09) received the B.S., M.S. and Ph.D degree from Beijing University of Posts\&Telecommunications (BUPT), China, in 1996, 2000 and 2005, respectively, where he is currently associate professor. He worked as a researcher in the companies including Siemens, Orange Labs R\&D (Beijing), China. His current research interests lie in the field of wireless communications, with emphasis on cooperative communication and heterogeneous networks

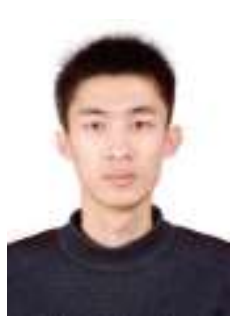

Wei Li received his B.S. degree from Shangdong Universit, China, in 2010. He is currently a candidate for M.S. in the Key Lab of Universal Wireless Communications, Ministry of Education, BUPT. His research interests include performance analysis of wireless networks.

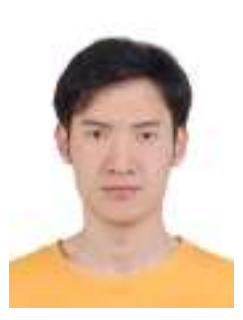

Fei Liu received his B.S. degree from the School of Information and Communication Engineering, Beijing University or Posts and Telecommunications (BUPT), China, in 2010. He is currently a candidate for M.S. in the Key Lab of Universal Wireless Communications, Ministry of Education, BUPT. His research interests include performance analysis of wireless networks, resource allocation and scheduling algorithm.

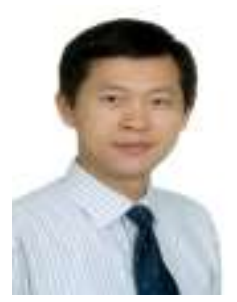

Wei Xiang (S'00-M'04-SM'10) received the B.Eng. and M.Eng. degrees, both in electronic engineering, from the University of Electronic Science and Technology of China, Chengdu, China, in 1997 and 2000, respectively, and the Ph.D. degree in telecommunications engineering from the University of South Australia, Adelaide, Australia, in 2004. Since January 2004, he has been with the Faculty of Engineering and Surveying, University of Southern Queensland, Toowoomba, Australia, where he was first an Associate Lecturer in Computer Systems Engineering from 2004 to 2006, then a Lecturer from 2007 to 2008, and currently holds a faculty post of Senior Lecturer. In 2008, he was a visiting scholar at the School of Electrical and Electronic Engineering, 
Nanyang Technological University, Singapore. During Oct. 2010 and Mar. 2011, he was a visiting scholar at the University of Mississippi, Oxford, MS, USA. He received the Best Paper Award at 2011 IEEE WCNC. He was named a Queensland International Fellow (2010-2011) by the Queensland Government of Australia, an Endeavour Research Fellow (2012-2013) by the Commonwealth Government of Australia, and a Smart Futures Fellow (2012-2015) by the Queensland Government of Australia. His research interests are in the broad area of communications and information theory, particularly coding and signal processing for multimedia communications systems. 